$34(14 \%)$ patients required tube replacement either due to tube damage or accidental removal. After clinical improvement and demonstration of normal swallow study, 168(71\%) patients had their tube removed after an average duration of 120 days. 68(29\%) of patients died due to non-PEG related complications during follow up.

Conclusions PEG is a safe and highly efficient feeding method in carefully selected patients with a low rate of complications and a high level of acceptance. It helps in the improvement of nutritional status and quality of life.

\section{IDDF2020-ABS-0105 DETAILED DIAGNOSTIC CLASSIFICATION FOR CROHN'S DISEASE CASES WITH ANAL FISTULA BASED ON ETIOLOGY BY EXAMINATION UNDER ANESTHESIA}

${ }^{1}$ Tsutomu Masuda*, ${ }^{2}$ Naoki Inatsugi, ${ }^{2}$ Syusaku Yoshikawa, ${ }^{2}$ Hideki Uchida, ${ }^{2}$ Takeshi Nakao, ${ }^{2}$ Hidenori Kashizuka, ${ }^{2}$ Kentaro Yamaoka, ${ }^{2}$ Mlzumi Inagaki. I'Ikoma Gastrocoloproctology Clinic, Japan; ${ }^{2}$ Nara Coloproctology Center, Japan

\subsection{6/gutjnl-2020-IDDF.82}

Background According to the etiological classification, anal fistulas in Crohn's disease (CD) which derived from CD lesions are secondary lesions (s.l.). We classified s.l. as follows: In case of presence of clear CD lesion at the primary orifice ( $p$. o.), it was classified as s.l.CD lesion $(+)$, whereas in the absence of CD lesion, it was classified as a s.l.CD lesion (-).

To examine the prognosis of patients that primary orifice (p.o.) is not on the dentate line and no clear CD lesion.

Methods 17 patients who have been followed up for $>5$ years post-the first examination under anesthesia (EUA) were included in the study.

To determine the therapeutic effect, we defined cases with closed fistula as cured, those in whom drain can be removed, but symptoms may exist as improved, those in whom drains cannot be removed as unchanged, and those in whom rectal amputation was required as worsened. The target cases were divided into s.l.CD lesion (+), and s.l.CD lesion (-), and the prognosis was examined.

Results Nine and eight patients showed s.l.CD lesion (+), and s.l.CD lesion (-), respectively. The results are shown in the table 1. Among s.l. cases, more patients with CD lesion (-) underwent biotreatment before EUA than those with s.l.CD lesion (+); furthermore, biotreatment was started earlier after EUA in the former than in the latter. Nevertheless, the prognosis of CD lesion (-) cases tended to be poor.

\begin{tabular}{lll} 
Abstract IDDF2020-ABS-0105 Table 1 & \\
\hline & $\begin{array}{l}\text { s.I.CDI(+) } \\
(\mathbf{n}=9)\end{array}$ & $\begin{array}{l}\text { s.I.CDI(-) } \\
(\mathbf{n}=8)\end{array}$ \\
\hline Age: (Mean \pm SD) (years) & $32.4 \pm 14.5$ & $31.6 \pm 9.2$ \\
sex: M/F & $5 / 4$ & $6 / 2$ \\
Anal fistula type: simple/cmplex & $0 / 9$ & $0 / 8$ \\
anal or rectal stenosis at EUA: yes/no & $1 / 8$ & $2 / 6$ \\
Pre EUA bio treatment: yes/no & $3 / 6$ & $4 / 4$ \\
Duration from EUA to start of bio treatment (mean $\pm S D)$ (month) & $13.3 \pm 22.7$ & $9.3 \pm 6.1$ \\
Post-EUA observation period (Mean $\pm S D)$ (month) & $99 \pm 18.3$ & $95.3 \pm 9.6$ \\
Therapeutic effect:cured/improved/unchanged/worse & $4 / 1 / 4 / 0$ & $2 / 1 / 4 / 1$ \\
\hline
\end{tabular}

Conclusions Based on the findings, the prognosis of patients that p.o. is not on the dentate line and no clear CD lesion is poor.

\section{IDDF2020-ABS-0111 DOES FRAILTY PREDICT POSTOPERATIVE OUTCOMES IN GERIATRIC PATIENTS RECEIVING SURGERY FOR COLORECTAL CANCER? A SYSTEMATIC REVIEW AND META-ANALYSIS}

${ }^{1}$ Xiaobo Yang* ${ }^{1}$ Chenying Xu, ${ }^{1}$ Jiumei Cao, ${ }^{2} \mathrm{Li}$ Xie, ${ }^{3}$ Qingqing Qiu, ${ }^{1}$ Jiaan Hu, ${ }^{4}$ Lifen $Y u$ ${ }^{5}$ Jean Woo. ${ }^{1}$ Department of Geriatrics, Ruijin Hospital, Shanghai Jiao Tong University School of Medicine, China; ${ }^{2}$ Clinical Research Institute, Shanghai Jiao Tong University School of Medicine, China; ${ }^{3}$ Department of Surgery, Ruijin Hospital Luwan Branch, Shanghai Jiao Tong University School of Medicine, China; ${ }^{4}$ Department of Gastroenterology, Ruijin Hospital, Shanghai Jiao Tong University School of Medicine, China; ${ }^{5}$ Department of Medicine and Therapeutics, Faculty of Medicine, The Chinese University of Hong Kong, Hong Kong

\subsection{6/gutjin-2020-IDDF.83}

Background Surgery remains the mainstay of colorectal cancer (CRC) and substantially reduces cancer-related morbidity and mortality. Frailty is defined as a biological syndrome, reflecting a state of impaired homeostatic reserve and predisposing to rehospitalisations and deaths in older individuals. Preoperative assessment for frailty is critically important in risk stratification and clinical decision-making. In this systematic review and meta-analysis, we aimed to quantitatively summarise the effect of frailty on postoperative outcomes in geriatric patients receiving surgery for CRC.

Methods A systematic literature search was conducted in MEDLINE, Cochrane and EMBASE from inception to 30 April 2020. Fully published articles reporting risk estimate (s) of frailty on postoperative complication(s), readmission and/or mortality in patients aged $\geq 65$ years who received surgery for CRC were eligible for qualitative and quantitative analyses.

Results Across 10 articles of 9 unique studies ( $n=69332$ ) that were eventually included in the systematic review and meta-analysis, overall prevalence of frailty was $23.0 \% \quad(95 \%$ CI: $11-43 \%, I^{2}=100 \%$ ). Odds ratios (ORs) on overall and severe postoperative complications were respectively increased by $2.36-\left(95 \% \mathrm{CI}: 1.66-3.35, P<0.01 ; I^{2}=12 \%\right)$ and 2.35 fold (95\% CI: $1.30-4.27, P<0.01 ; I^{2}=72 \%$ ) in frail patients compared to non-frail counterparts. On pooled analysis, frailty was significantly associated with an increased risk of postoperative readmission (OR:1.91; 95\% CI: 1.35-2.70, P<0.01; $I^{2}$ $=6 \%)$. Whilst a significantly higher risk of frailty on mortality during 12 months after CRC surgery was observed (OR: 5.52; 95\% CI:4.40-6.92, $\left.P<0.01 ; I^{2}=89 \%\right)$, the summary OR on 30-day/inpatient mortality crossed the null line (OR: 1.65; 95\% CI: $\left.0.56-4.93, P=0.37 ; I^{2}=55 \%\right)$. Funnel plot and Duval-Tweedie's trim and fill test did not reveal significant publication bias.

Conclusions In the studies reviewed, frailty appeared to be associated with increased risks for postoperative complications, readmission and mortality during 12 months in patients aged $\geq 65$ years who received surgery for CRC. Nevertheless, no significant association between frailty and 30-day/inpatient mortality was observed. 